Journal of Engineering and Applied Sciences 14 (12): 4158-4163, 2019

ISSN: 1816-949X

(C) Medwell Journals, 2019

\title{
Face Recognition using Hybrid Techniques
}

\author{
Asaad Noori Hashim and Nedaa Kream Shalan \\ Department of Computer Science, Faculty of Computer Science and Mathematics, \\ University of Kufa, Najaf, Iraq
}

\begin{abstract}
As one of the more effective applications that used in image understanding and analysis, face recognition has given significant attention in last years. There are several methods used for face recognition such as PCA, LDA, Zernike and each method has limitations and strength points. This study offers a statistical estimate of the execution to recognized the human faces in digital images by using a new feature extraction method which based on hybrid system for face recognition that contains: Gabor filters and singular value decomposition. By using the Gabor filters 40 sub-images were obtained from the original images in 5 scales and 8 orientations and by SVD using one matrix U from 3 matrices [USV] that have singular value which represent feature extracted from an image and using hybrid technique normalize features vectors by Z-score to get optimal values then fusion two features vectors $2 \mathrm{D}$ Gabor filter and SVD to optimize the recognition rate. Finally, classification step is done by (Euclidean distance) to take the decision about matching. The outcomes experimental showed that the suggest system is effective, it has been tested using ORL face images databases with 10 cases and achieved recognition rate from $77.7-100 \%$, also, applied on FEI Brazil face database with 5 cases and achieved recognition rate from to $84-100 \%$.
\end{abstract}

Key words: Face recognition, Gabor filter, singular value decomposition, database, recognition rate, scales

\section{INTRODUCTION}

Face reorganization is consider as aspect of biomedical technology that is serviceable for the a lot off applications like in banking, human computer interaction, implementation security and retrieval of data base, personal identification passports, driver licenses, law enforcement, surveillance system, etc. (Reddy and Raju, 2016). The challenges in face recognition are orientations, different poses, expressions, illuminations and wears the glasses, likewise, As yet, face recognition algorithms are faced many challenges of recognizing face image under fickle environmental bands (Anonymous, 2012).

For this cause, system involves extract features from an images and then matched it, regardless of changing in lighting, ageing, occlusion, changing in expression. Generally, face recognition system can be used in two ways as follow.

Verification: (1-to-1) which include the state where the identity of a single individual has to be confirmed.

Identification: (1-to-many) which is used when an image is given and the identity of the individual is to be specified from an ready database by comparing to with all available images (Barnouti et al., 2016).
Over the last two centuries, face recognition has fall a significant topic for many researchers. It has establish applications mostly in computer vision, phscyhology and neuroscience (Malekan, 2015). The fast developments in face recognition has been assisted by developments in algorithms and diverse (hybrid) algorithms for reach to new methods with more accurate results and less cost. Face recognition methods are divided to two kinds structural (texture) and statistical approaches (Hashim and Hussain, 2014). In this study, we using 2D Gabor/SVD algorithms for features extraction as a new method for hybrid face recognition that used statistical and structural system. In the latest years, Gabor filter has given great important and has appear as one of the most common kinds to texture feature extraction, owing to its using of optimal localization in both spatial and frequency domains. Also, Gabor wavelet-based face representation has demonstrated its robustness against variations such as illumination, scale, translation and rotation (Cho et al., 2014). The SVD in the digital applications gives robust/strong technique, also, it considered more manageable, hence, it used in the data retrieval for minimizing document dimension vector space (Jain and Kumar, 2016) and it used to extract features from image. For several years, face recognition has acquired important attention at many researcher fields. So, we introduce

Corresponding Author: Asaad Noori Hashim, Department of Computer Science, Faculty of Computer Science and Mathematics, University of Kufa, Najaf, Iraq 
overview of some methods that considered as well-known algorithms for human face recognition. Ouanan et al. (2015a, b) presented a new feature extractor-Fusion scheme using two powerful feature descriptor known as Gabor Filters (GFs) and Histogram of Oriented Gradient (HOG), they filtered the faces images with multi scale and multi resolution of Gabor filter bank for generate multiple Gabor Magnitude Images (GMIs) finally, the down-sampled GMIs and implemented histogram of oriented gradient to compose the features. Asiedu et al. $(2015,2016)$ proposes multivariate statistical estimation of the recognition achievement for (PCA/SVD), first with (whitened PCA/SVD) under different environmental constraints and second using Principal Component Analysis (PCA) with Singular Value Decomposition(SVD) and using fast Fourier Transform for preprocessing steps (FFT-PCA/SVD). Barnouti et al. (2016) used PCA with LDA for extracting features and reducing dimensions of images, also, detecting and cropping faces in database using (Viola-Jones) method while measured the distance between two images by Square Euclidean distance. Ouanan et al. (2015a, b) invented a novel face recognition framework based on an efficiently combination of local and global features extracted using Gabor and Zernike with SVM classifier. Rahul Jain and Sanjay Kumar are upgrade the precision of the face reorganization system that using PCA with SVD based algorithm. Ahmmad et al. (2016) introduced a texture feature extraction method using a 2D log Gabor filter with the singular value decomposition algorithm effects to extract appropriate distinctive texture information as well as Naive Bayes as classifier. Dabhade et al. (2016), suggested a hybrid face recognition system that uses Gabor with Kernel Principle Component Analysis (KPCA) feature-based algorithms and Mahalabonis distance on Hong Kong PolyU database. Barrah et al. (2017) introduced a face recognition method that used SVD with 3D Local Binary Pattrns (3DLBP) to encode correlative features of points on facial surface.

\section{MATERIALS AND METHODS}

The proposed system: The proposed methodology is employed to extract human face features using Gabor filters and singular value decomposition from images in parallel way. Then normalize each one using Z-score after that, the two vectors of features are Fusion by concatenated them to became one vector. The proposed system has five stages: preprocessing, features extraction,

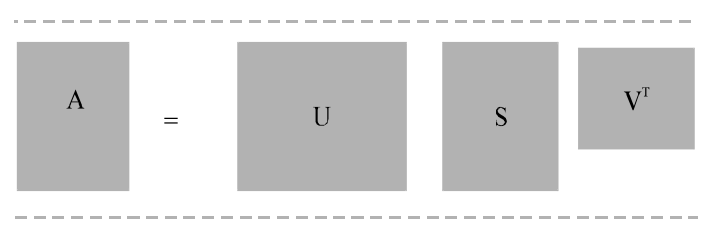

Fig. 1: Illustration of factoring

normalization, fusion and matching using Euclidean distance. The study utilized two sets of face database to prove accurate and efficiency of the proposed system, the AT\&T database (ORL) and Brazilian database, Fig. 1 and 2 shows diagram for the proposed system.

Preprocessing: In this step, the images are converted into gray scale, doubled and resized to quadratic array as $(64 \times 64)$.

Features extraction: In this step, the features are extracted from images twice in parallel way, first using $2 \mathrm{D}$ Gabor wavelet filter, second using singular value decomposition.

Singular value decomposition: It reflect a fundamental role in many different applications such face recognition, object detection, image compression signal processing, texture classification, etc., the remarkable feature of SVD is that it can be implemented on any real matrix. Once SVD has identified the most variation, it is possible to find the best approximation of the original data points using fewer dimensions (Asiedu et al., 2015). The singular value decomposition of a rectangular matrix. $\mathrm{A}$ is a decomposition of the form:

$$
\mathrm{A}=\mathrm{USV}^{\mathrm{T}}
$$

Where:

$\mathrm{A}=$ An matrix with $(\mathrm{m} \times \mathrm{n})$ dimensional

$\mathrm{U}=\mathrm{m} \times \mathrm{m}$

$\mathrm{V}=\mathrm{n} \times \mathrm{n}$

$\mathrm{U}$ and $\mathrm{V}$ are considered orthogonal matrices and $\mathrm{S}$ is an $\mathrm{m} \times \mathrm{n}$ and consider diagonal matrix (Asiedu et al., 2015). That is $U$ are computed as the eigen vector of $\left(\mathrm{AA}^{\mathrm{T}}\right)$ and $\mathrm{V}$ are computed as the eigen vector of $\left(\mathrm{A}^{\mathrm{T}} \mathrm{A}\right)$ that denoted as:

$$
\begin{aligned}
& \mathrm{AA}^{\mathrm{T}}=\mathrm{USV}^{\mathrm{T}}\left(\mathrm{USV}^{\mathrm{T}}\right)^{\mathrm{T}}=\mathrm{USV}^{\mathrm{T}} \mathrm{VSU}^{\mathrm{T}}=\mathrm{US}^{2} \mathrm{U}^{\mathrm{T}} \\
& \mathrm{A}^{\mathrm{T}} \mathrm{A}=\left(\mathrm{USV}^{\mathrm{T}}\right)^{\mathrm{T}} \mathrm{USV}^{\mathrm{T}}=\mathrm{VSU}^{\mathrm{T}} \mathrm{USV}^{\mathrm{T}}=\mathrm{VS}^{2} \mathrm{~V}^{\mathrm{T}}
\end{aligned}
$$




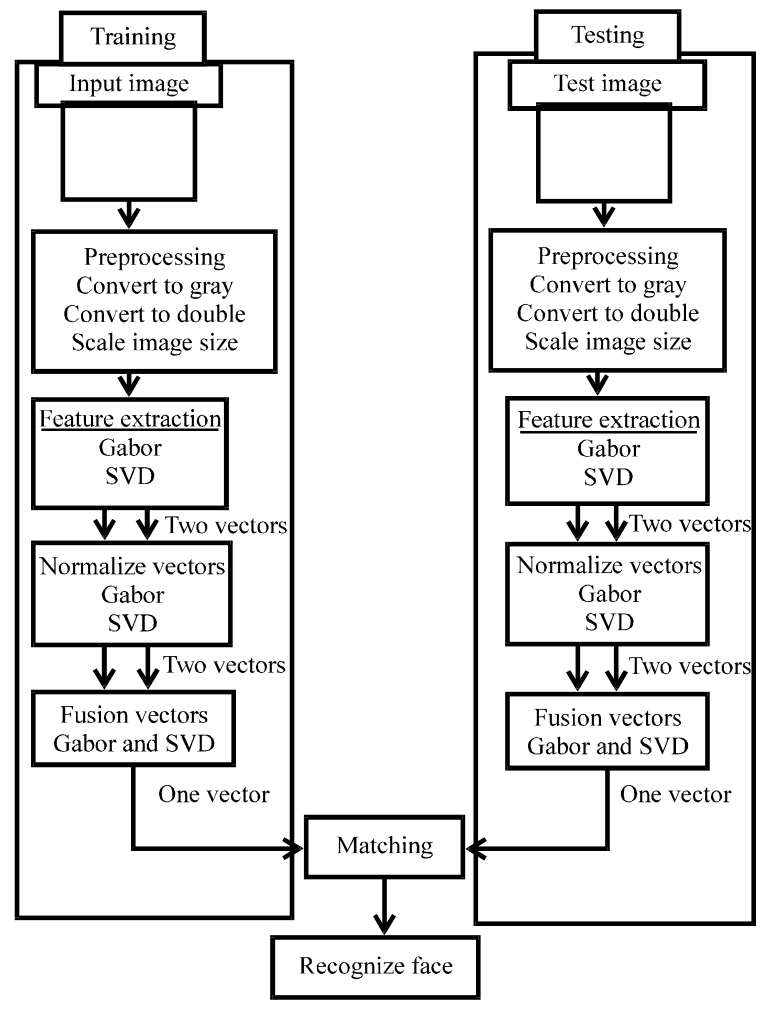

Fig. 2: Explain the main steps of the proposed hybrid system

Gabor filter: The Gabor filter was first proposed by Dennis Gabor in 1946. The Gabor wavelet kernel function has similar characteristics as the reflection area of the human cerebral cortex. In denoted the spatial domain, a two dimensional Gabor filter is composed from a Gaussian kernel function adjusted by a sinusoidal plane wave is indicated as follows (Thangairulappan and Jeyasingh, 2012):

$$
\begin{gathered}
\varphi \mathrm{u}, v=\varphi_{\Pi}(\mathrm{f}, \theta, \gamma, \eta)(\mathrm{x}, \mathrm{y})= \\
\mathrm{f}^{2} / \pi \gamma \eta \exp \left(-\left(\alpha^{2} \mathrm{x}^{2}+\beta^{2} \gamma^{\prime 2}\right)\right) \exp \left(\mathrm{j} 2 \pi \mathrm{fx} \mathrm{x}^{\prime}\right) \\
\mathrm{x}^{\prime}=\mathrm{x} \cos \theta+\mathrm{y} \sin \theta \\
\mathrm{y}^{\prime}=-\mathrm{x} \sin \theta+\mathrm{y} \cos \theta
\end{gathered}
$$

where, f represent the central frequency of the sinusoidal plane wave while is refer to the anti-clockwise rotation of the Gaussian and the plane wave, also, "is denoted the sharpness of the Gaussian along the major axis parallel to the wave and ${ }_{\beta}$ is the sharpness of the Gaussian minor axis perpendicular to the wave:

$$
\gamma=\frac{\mathrm{f}}{\alpha}
$$

And:

$$
\eta=\frac{f}{\beta}
$$

This hold over the ratio between frequency and sharpness constant. As well to extract features from a face image, a set of Gabor filters with different frequencies and orientations are required as follows:

$$
\begin{gathered}
f_{u}=f_{\max } / \sqrt{2^{u}} \\
\theta_{v}=\frac{v}{8} \pi
\end{gathered}
$$

Where:

$$
\begin{aligned}
\mathrm{u} & =0, \ldots, \mathrm{U}-1 \\
& =0, \ldots, \mathrm{V}-1 \\
\mathrm{f}_{\max } & =\text { Refer to highest peak frequency } \\
\mathrm{U} & =\text { The number of scales } \\
\mathrm{V} & =\text { The number of orientations }
\end{aligned}
$$

As a result of the complex exponential, the filter has both real and imaginary parts (Ahmmad et al., 2016). Depended on the requirements of application the system determined number of Gabor filters that used for feature extraction from an image. The Gabor representation of a face image $\mathrm{I}(\mathrm{x})$ can be obtained by convolving the image with the Gabor filters that referred as (GWR) 'Gabor Wavelet Representation' as (Cho et al., 2014):

$$
\mathrm{G}_{\mathrm{u}, \mathrm{v}}(\mathrm{x})=\left(\mathrm{I}^{*} \varphi_{\mathrm{u}, \mathrm{v}}\right) *(\mathrm{x})
$$

where $G_{u}$, (x) refers to results of the convolution of the Gabor filter at orientation $\mathrm{u}$ and with scale . where, $\left(^{*}\right)$ denotes the convolution operation.

$\mathrm{G}_{\mathrm{u},},(\mathrm{x}) \mathrm{u}=0, \ldots, 4, v=0, \ldots, 7$, a filter bank consisting of Gabor filters with various scales and rotations is created (Dabhade et al., 2016) (Fig. 3). Refers to the combination of multiple features of the same individual. The Fusion method of features vectors results to the improvement of the performances by reducing the an accept results, Fusion has been used effectively for years in large scale automatic many recognition system with three type (fusion at the feature extraction step, fusion at the matching scores step and at the decision step) (Kaur and Neeru, 2015). Before fusion two features vectors must normalize each one in this proposed we used Z-score normalization as follow: 


$$
\mathrm{Z} \text {-score }=\frac{(\text { Feature vector }) \text {-Mean }(\text { Feature vector })}{\text { Standard devation(Feature vector })}
$$

Matching: In this study, we used a Euclidean distance measure for matching. The recognition rate and error rate of proposed system is calculated by the following equation and report in our experiments:

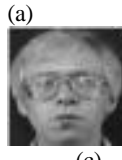

(b)

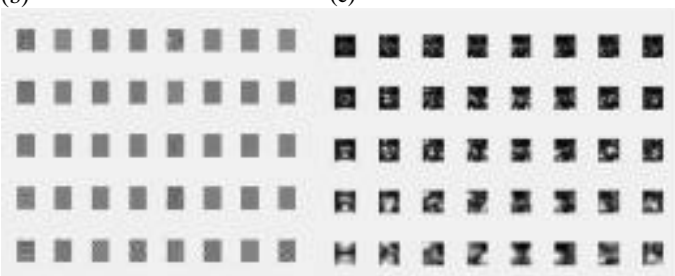

Fig. 3: a) Face image; b) Gabor filter response (magnitude) images and c) Gabor filter response (real) images
Recognition rate $=\frac{\text { No. of recognized samples }}{\text { Total number of samples }} \times 100 \%$

Recognition error rate $=\frac{\text { No. of non recognized samples }}{\text { Total number of samples }} \times 100 \%$

\section{RESULTS AND DISCUSSION}

In this study, we have presented a novel face recognition system using hybrid technique that combine 2D Gabor and SVD, the performance with SVD alone is $71.3-97.5 \%$ with AT\&T and from $71.5-95 \%$ with FEI while the recognition with Gabor alone gained rate 76.6-97.5\% with AT\&T, also, 72.5-95\% with FEI but the best results are obtained using Fusion both methods and we noted that the higher recognition rate with lower error ratio using hybrid system that achieved 77.7-100\% with AT\&T, also, 84-100\% with FEI. Generally, the results shown in Table 1 and 2 and Fig. 4.

Table 1: Results of appling proposed algorithm to ORL data set

\begin{tabular}{|c|c|c|c|c|c|c|}
\hline Cases (Testing-training) & Poses for testing & No. of testing & Poses for training & No. of training & Recognition error (\%) & Recognition rate $(\%)$ \\
\hline Case (1-9) & 1 & 40 & $2-10$ & 360 & 0.00 & 100.00 \\
\hline Case $(2-8)$ & $1-2$ & 80 & $3-10$ & 320 & 1.25 & 98.75 \\
\hline Case (3-7) & $1-3$ & 120 & $4-10$ & 280 & 1.66 & 98.30 \\
\hline Case (4-6) & $1-4$ & 160 & $5-10$ & 240 & 1.87 & 98.12 \\
\hline Case (5-5) & $1-5$ & 200 & $6-10$ & 200 & 2.00 & 98.00 \\
\hline Case (5-5) swap & $6-10$ & 200 & $1-5$ & 200 & 5.00 & 95.00 \\
\hline Case (6-4) & $5-10$ & 240 & $1-4$ & 160 & 4.58 & 95.41 \\
\hline Case (7-3) & $4-10$ & 280 & $1-3$ & 120 & 10.00 & 90.00 \\
\hline Case (8-2) & $3-10$ & 320 & $1-2$ & 80 & 13.12 & 86.87 \\
\hline Case $(9-1)$ & $2-10$ & 360 & 1 & 40 & 22.22 & 77.77 \\
\hline
\end{tabular}

Table 2: Results of appling proposed algorithm to FEI data set

\begin{tabular}{lcccccc}
\hline Cases (Testing-training) & Poses for testing & No. of testing & Poses for training & No. of training & Recognition error (\%) Recognition rate (\%) \\
\hline Case (1-9) & 1 & 40 & $2-10$ & 360 & 0.00 & 100.00 \\
Case (2-8) & $1-2$ & 80 & $3-10$ & 320 & 2.50 & 97.50 \\
Case (3-7) & $1-3$ & 120 & $4-10$ & 280 & 3.33 & 96.66 \\
Case (4-6) & $1-4$ & 160 & $5-10$ & 240 & 11.3 & 88.75 \\
Case (5-5) & $1-5$ & 200 & $6-10$ & 200 & 16.0 & 84.00 \\
\hline
\end{tabular}

Table 3: Result of all cases applied on FEI and AT\&T face database

\begin{tabular}{lcccccc}
\hline Database/cases & $\begin{array}{c}\text { Gabor recognition } \\
\text { rate (\%) }\end{array}$ & $\begin{array}{c}\text { Gabor } \\
\text { error rate (\%) }\end{array}$ & $\begin{array}{c}\text { SVD recognition } \\
\text { rate (\%) }\end{array}$ & $\begin{array}{c}\text { SVD error } \\
\text { rate (\%) }\end{array}$ & $\begin{array}{c}\text { Hybrid recognition } \\
\text { rate (\%) }\end{array}$ & $\begin{array}{c}\text { Hybrid error } \\
\text { rate (\%) }\end{array}$ \\
\hline AT\& T & & & & & & \\
Case(1-9) & 97.50 & 2.50 & 97.50 & 2.50 & 100.00 & 0.00 \\
Case(2-8) & 98.75 & 1.25 & 98.75 & 1.25 & 98.75 & 1.25 \\
Case(3-7) & 99.16 & 0.83 & 98.30 & 1.66 & 98.30 & 1.66 \\
Case(4-6) & 98.75 & 1.25 & 96.80 & 8.12 & 98.12 & 1.87 \\
Case(5-5) & 97.50 & 2.50 & 91.50 & 9.00 & 98.00 & 2.00 \\
Case(5-5) swap & 93.00 & 7.00 & 91.00 & 10.83 & 95.00 & 5.00 \\
Case(6-4) & 93.30 & 6.66 & 89.10 & 13.92 & 90.00 & 10.00 \\
Case(7-3) & 86.78 & 13.21 & 86.07 & 17.50 & 86.87 & 13.12 \\
Case(8-2) & 88.12 & 11.87 & 82.50 & 28.70 & & 77.77 \\
Case(9-1) & 76.60 & 23.40 & 71.30 & & & 22.22 \\
\hline
\end{tabular}


Table 3: Continue

\begin{tabular}{lccccrr}
\hline Database/cases & $\begin{array}{c}\text { Gabor recognition } \\
\text { rate (\%) }\end{array}$ & $\begin{array}{c}\text { Gabor } \\
\text { error rate (\%) }\end{array}$ & $\begin{array}{c}\text { SVD recognition } \\
\text { rate (\%) }\end{array}$ & $\begin{array}{c}\text { SVD error } \\
\text { rate (\%) }\end{array}$ & $\begin{array}{c}\text { Hybrid recognition } \\
\text { rate (\%) }\end{array}$ & $\begin{array}{c}\text { Hybrid error } \\
\text { rate (\%) }\end{array}$ \\
\hline FEI & & & & & & \\
Case(1-9) & 95.00 & 5.00 & 95.00 & 5.00 & 100.00 & \\
Case(2-8) & 92.50 & 7.50 & 91.25 & 8.75 & 97.50 & 0.00 \\
Case(3-7) & 93.33 & 6.66 & 90.00 & 10.00 & 96.66 & 3.50 \\
Case(4-6) & 84.37 & 15.62 & 84.37 & 15.62 & 88.75 & 11.30 \\
Case(5-5) & 72.50 & 27.50 & 71.50 & 28.50 & 84.00 & 16.00 \\
\hline
\end{tabular}
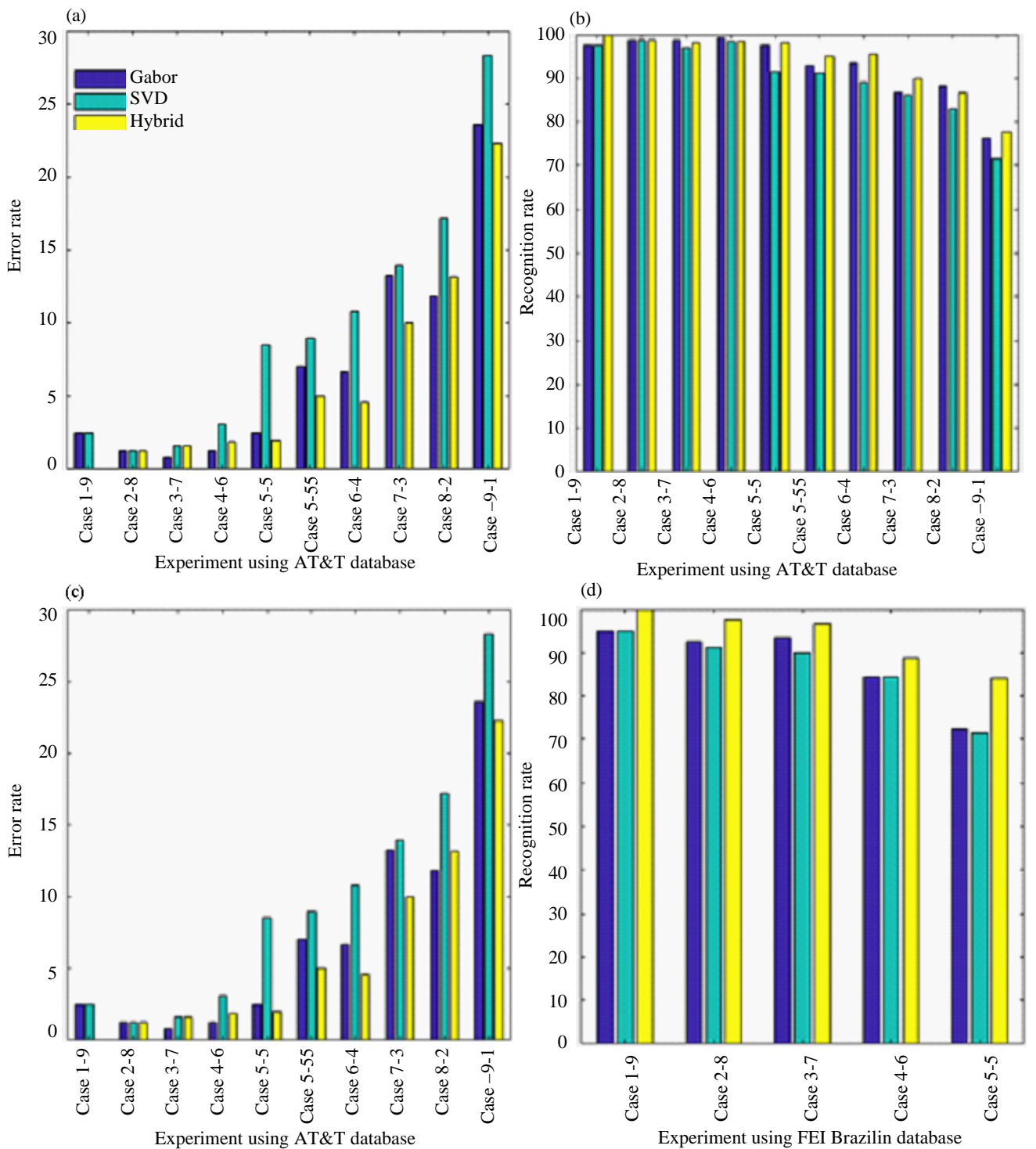

Fig. 4: a) Diagram show error rate of whole AT\&T database; b) Diagram show recognition rate of whole AT\&T database; c) Diagram show error rate of whole FEI database and d) Diagram show recognition rate of whole FEI T database

\section{CONCLUSION}

The SVD has problems with rotation while Gabor is invariant to rotation (Gabor have a good deal with arcs).
The SVD have strength in analysis of unique value of an array of image. The first five poses of AT\&T is easier to recognition if it compared with second five poses (notice in case 5,6 with swap poses). 
Also notice that, the statistical measures are tricky (sometimes) and it has random behavior, so with increasing training sets, the default case there is increasing in recognition ratio but this don't happened always. Table 3 show the results of all cases applied on AT\&T and FEI Brazilian face database.

\section{REFERENCES}

Ahmmad, R.S., R.A. Rab and N.S. Anjum, 2016. 2D $\log$ gabor and SVD based parallel texture feature extraction using NVIDIA GPU. Ph.D Thesis, BRAC University, Dhaka, Bangladesh.

Anonymous, 2012. The FEI face database is available for research purposes only: Permission to use but not distribute or reproduce this database is granted to all researchers. International Federation for Equestrian, Lausanne, Switzerland.

Asiedu, L., A. Adebanji, F. Oduro and F.O. Mettle, 2015. Statistical evaluation of face recognition techniques under variable environmental constraints. Intl. J. Stat. Probab., 4: 93-111.

Asiedu, L., A. Adebanji, F.T. Oduro and F.O. Mettle, 2016. Statistical assessment of PCA/SVD and FFT-PCA/SVD on variable facial expressions. Br. J. Math. Comput. Sci., 12: 1-23.

Barnouti, N.H., S.S.M. Al-Dabbagh, W.E. Matti and M.A.S. Naser, 2016. Face detection and recognition using viola-jones with PCA-LDA and square euclidean distance. Intl. J. Adv. Comput. Sci. Appl., 7: 371-377.

Barrah, E.M., R. Ahdid, S. Safi and A. Malaoui, 2017. 3DLBP and SVD fusion for 3D face recognition using range image. Comput. Sci. Inf. Technol., 5: 61-65.
Cho, H., R. Roberts, B. Jung, O. Choi and S. Moon, 2014. An efficient hybrid face recognition algorithm using PCA and GABOR wavelets. Intl. J. Adv. Rob. Syst., 11: 59-66.

Dabhade, S.B., N.S. Bansod, Y.S. Rode, M.M. Kazi and K.V. Kale, 2016. Hyper spectral face recognition using KPCA. Intl. J. Sci. Eng. Res., 7: 548-550.

Hashim, A.N. and Z. Hussain, 2014. Local and semi-global feature-correlative techniques for face recognition. Int. J. Adv. Comput. Sci. Applic., 5: 157-167.

Jain, R. and S. Kumar, 2016. High accuracy face reorganization by PCA-SVD. Intl. J. Eng. Sci. Res. Technol., 2016: 6-10.

Kaur, P. and E.N. Neeru, 2015. A hybrid approach for secure biometric authentication using fusion of iris and ear. Intl. J. Adv. Res. Comput. Sci. Software Eng., 5: 438-445.

Malekan, M., 2015. Face recognition based on local Zernike moments. Master Thesis, Eastern Mediterranean University, Famagusta, Northern Cyprus.

Ouanan, H., M. Ouanan and B. Aksasse, 2015b. Gabor-HOG features based face recognition scheme. Telkomnika Indonesian J. Electr. Eng., 15: 331-335.

Ouanan, H., M. Ouanan and B. Aksasse, 2015a. Gabor-Zernike features based face recognition scheme. Int. J. Imaging Rob., 16: 118-131.

Reddy, K.S.P. and K.N. Raju, 2016. Design and implementation of an algorithm for face recognition by using Principal Component Analysis (PCA) in MATLAB. Intl. J. Adv. Res. Comput. Sci. Software Eng., 6: 115-119.

Thangairulappan, K. and J.B.V. Jeyasingh, 2012. Face representation using combined method of gabor filters, wavelet transformation and DCV and recognition using RBF. J. Intell. Learn. Syst. Appl., 4: 266-273. 